\title{
Brain atrophy and cognitive deficits in Cushing's disease
}

\author{
Chirag G. Patil, M.D., Shivanand P. Lad, M.D., Ph.D., Laurence Katznelson, M.D., \\ AND EDWARD R. LAWS, JR., M.D. \\ Department of Neurosurgery, Stanford University School of Medicine, Stanford, California
}

\begin{abstract}
$\checkmark$ Cushing's disease is associated with brain atrophy and cognitive deficits. Excess glucocorticoids cause retraction and simplification of dendrites in the hippocampus, and this morphological change probably accounts for the hippocampal volume loss. Mechanisms by which glucocorticoids affect the brain include decreased neurogenesis and synthesis of neurotrophic factors, impaired glucose utilization, and increased actions of excitatory amino acids. In this review, the timing, pathology, and pathophysiology of the brain atrophy in Cushing's disease are discussed. The correlation of atrophy with cognitive deficits and its reversibility is also reviewed. (DOI: 10.3171/FOC-07/09/E11)
\end{abstract}

\section{KEY WORDS - brain atrophy - Cushing's disease - cognitive impairment • glucocorticoids • hippocampal atrophy}

$\mathrm{C}$ USHING's disease results from sustained pathological hypercortisolism secondary to excessive adrenocorticotropin hormone secretion by tumors of the pituitary gland. Clinical features of Cushing's disease such as abnormal fat distribution, wide violaceous striae, hirsutism, impaired glucose tolerance, and osteoporosis are often discussed and investigated. The experienced clinician, however, is very aware of the rarely discussed, but often disabling, cognitive deficits and emotional symptoms that accompany Cushing's disease. In this review, we discuss the neurobiological basis of these impairments.

We previously documented a striking radiographic feature of pathological hypercortisolism, namely the marked cortical and subcortical brain atrophy seen in patients with Cushing's disease (Fig. 1). ${ }^{19}$ Brain atrophy, especially in the hippocampus, has been well documented in both adult and pediatric patients treated with exogenous glucocorticoid therapy. $1,2,5,6,16,21$ In this review, the timing, pathology, and pathophysiology of glucocorticoid-induced brain atrophy are discussed. In addition, the limited information specific to hippocampal atrophy, cognitive impairments, and mood disorders in Cushing's disease is presented.

\section{Effects of Glucocorticoids on Hippocampal and Brain Volume}

Animal Models

Rodent and primate animal models have been developed to investigate the effects of glucocorticoids on the hippocampus. Rodent studies have consistently demonstrated

Abbreviations used in this paper: EAA = excitatory amino acid; LTP $=$ long-term potentiation. that raising levels of corticosterone, the primary glucocorticoid in rodents, results in a decrease in hippocampal volume. ${ }^{20}$ Sapolsky and colleagues ${ }^{17}$ stereotactically implanted glucocorticoid and control (cholesterol) pellets in vervet monkeys' hippocampi and demonstrated hippocampal damage and volume loss after 1 year at postmortem examination. Exposure to excess glucocorticoids does not kill or reduce the number of pyramidal or glial cells in the hippocampus. ${ }^{26}$ Several early studies showed cell layer irregularities, soma shrinkage, and condensation of the pyramidal cells after excess glucocorticoid administration. ${ }^{17,30}$ Recent studies that involved rigorous quantitative methods, however, have not shown decreases in pyramidal cell diameter, condensation, or cell layer irregularities. ${ }^{6,26}$

What, then, accounts for the well-documented decreases in hippocampal volume? On a cellular level, exposure to excess glucocorticoids appears to alter the dendritic shape of the hippocampal pyramidal neurons. ${ }^{29,30}$ The apical dendrites in the CA3 region of the hippocampus decrease in length and show decreased branching. This decrease in apical dendritic neuropil volume (dendritic atrophy) appears to be responsible for the hippocampal volume loss. Decreases in mitochondrial volume have also been reported and may also contribute to hippocampal volume loss. ${ }^{6}$ Tata and associate $^{26}$ have demonstrated a profound loss of synapses in the CA3 region of the hippocampus, independent of the volume loss. This finding suggests that volume measures may significantly underestimate the effects of glucocorticoids on the brain.

Glucocorticoid receptors are widely distributed throughout the brain. The highest density of glucocorticoid receptors in the central nervous system is in the hippocampus. ${ }^{4}$ Coburn-Litvak and colleagues ${ }^{6}$ have demonstrated that chronic glucocorticoid exposure not only decreases hip- 


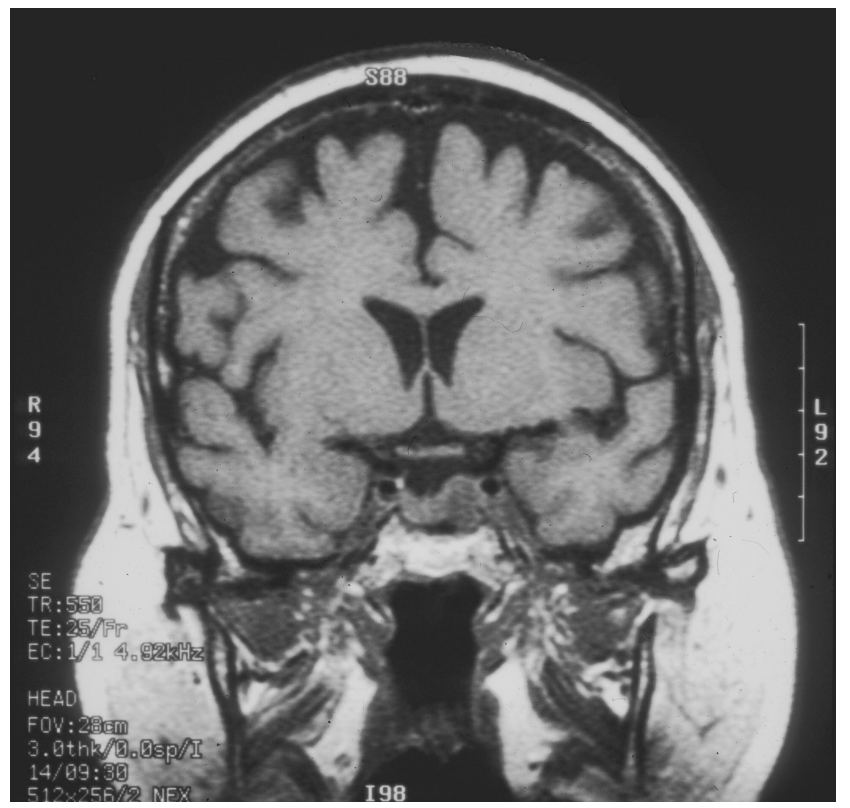

FIG. 1. Magnetic resonance image showing brain atrophy in a 32-year-old woman with Cushing's disease.

pocampal volume, but also reduces total brain weight. These data highlight the fact that the effects of glucocorticoids are not limited to the hippocampus-atrophy has been documented in the prefrontal cortex and other cortical areas. $^{1,12}$ It is also entirely possible that glucocorticoids affect specific areas of the brain differently. For example, in contrast to the dendritic atrophy observed in the hippocampus and prefrontal cortex, glucocorticoids increase dendritic growth in the amygdala. ${ }^{12}$ The significance of the effects of glucocorticoids on the amygdala, prefrontal cortex, and other areas of the brain awaits further investigation.

\section{Clinical Studies}

Brain and hippocampal atrophy have been documented in imaging studies of patients receiving glucocorticoid therapy for a variety of indications, including asthma, rheumatic diseases, and multiple sclerosis., ${ }^{1,2,14}$ Furthermore, endogenous elevations of plasma cortisol levels are a common trait in several pathological conditions, including Cushing's disease, major depression, and posttraumatic stress disorder. These disorders also share cognitive impairments such as memory deficits. Given the findings from animal and clinical studies, together with the fact that the hippocampus is critical in memory processing, it is logical to contend that excess glucocorticoids are responsible for changes in the hippocampus and consequently for the hippocampal-dependent memory impairment. Magnetic resonance imaging studies have documented a decrease in the hippocampal volume of patients with excess glucocorticoids, and shown correlations between this decrease and the presence of cognitive deficits. ${ }^{2,18,23}$

Cognitive impairments are common in patients with Cushing's disease and are correlated with elevated cortisol levels. Starkman and coworkers ${ }^{22}$ found cognitive deficits in several domains in patients with Cushing's disease, including verbal intellectual skills, learning, and memory.
The largest decline in cognitive function in this study was found in measures of the verbal intelligence quotient and verbal learning and recall. These impairments are consistent with the clinical cognitive complaints reported by patients with Cushing's disease. ${ }^{24}$ In contrast to dementia, delirium, and aging, which show increased vulnerability across visuospatial measures, verbal functions are most prominently affected in Cushing's disease. The deficits in verbal intellectual skills suggest involvement of the neocortex, whereas the impairments in verbal learning and recall are consistent with the increasingly accepted view that the hippocampus is especially vulnerable to the effects of glucocorticoids. . $^{172,27,29}$

Mood disorders, especially depression, are common in patients with Cushing's disease. Other psychiatric disturbances linked with excess glucocorticoids include anxiety, excitability, hypomania, and psychosis. Although atrophy of the prefrontal cortex has been linked with depression, anxiety and excitability have been correlated with increases in the size and activity of the amygdala. ${ }^{12,13}$ Therefore, the role of glucocorticoids in mediating the mood disturbances noted in patients with Cushing's disease needs to be elucidated further.

\section{Mechanisms of Glucocorticoid-Induced Brain Atrophy and Hippocampal Changes}

Glucocorticoids impair the ability of hippocampal neurons to survive various neurological insults, including hypoxia, ischemia, seizures, and exposure to antimetabolites. ${ }^{11,17,25}$ Mechanisms by which glucocorticoids induce morphological changes in the brain are largely unknown, although four theories (backed by limited evidence) have been suggested. These four theories are briefly summarized.

\section{Decreased Glucose Utilization}

Glucocorticoids decrease glucose uptake in the brain, and over time this may lead to brain atrophy. Glucocorticoids are known to affect cellular glucose metabolism and decrease glucose utilization in peripheral tissues and the brain. ${ }^{3,10}$ As reported by Brunetti and associates, ${ }^{3}$ the fluorodeoxyglucose positron emission tomography findings of a generalized reduction in cerebral glucose metabolism in all areas of the brain in patients with Cushing's disease support the reduced glucose utilization hypothesis.

\section{Increased Actions of EAA Neurotransmitters}

Excitatory amino acids such as glutamate are known to cause cell damage. There is some evidence to suggest that glucocorticoids may increase the release or enhance the effects of EAAs. Phenytoin, which may decrease the effects of EAAs, prevents the dendritic atrophy produced by excess glucocorticoids in the hippocampus. ${ }^{28}$ These findings support the role of glucocorticoid-induced EAAs in the origins of hippocampal and brain atrophy.

\section{Inhibition of LTP and Decrease in Neurotrophic Factors}

Long-term potentiation is believed to be the mechanism behind learning and formation of memories. Zhou and coworkers $^{31}$ showed that excess glucocorticoids inhibit 
LTP in rats. In addition, this study demonstrated that glucocorticoids reduce the synthesis of neurotrophic factors such as nerve growth factor- $\beta$ and brain-derived neurotrophic factor. The reduction of neurotrophic factors may be a presynaptic mechanism by which LTP is inhibited by glucocorticoids. In this theory, brain atrophy is explained by the reduction in neurotrophic factors, and the cognitive deficits are accounted for by the inhibition of LTP.

\section{Decreased Neurogenesis}

The subgranular layer of the dentate gyrus is involved in neurogenesis and gives rise to granular neurons. ${ }^{12}$ There is some evidence that stress and hormonal changes, such as excess glucocorticoids, may suppress neurogenesis in the dentate gyrus. ${ }^{7}$ This impaired neurogenesis could account for some of the hippocampal volume loss associated with glucocorticoids.

\section{Duration of Excess Glucocorticoid Exposure and Onset of Cognitive Deficits and Brain Atrophy}

Primate studies using exogenous glucocorticoids show that hippocampal changes are present within 1 year of glucocorticoid exposure. Because of the often insidious onset of symptoms in Cushing's disease, no specific data on exposure duration and brain atrophy are available. Clinical investigations of exogenous glucocorticoid therapy show an exposure duration of as short as 2 to 6 months before changes in the brain and hippocampus are detected., ${ }^{5,21}$ Cerebral cortical atrophy has been reported within 6 months of glucocorticoid exposure, even in children. ${ }^{21}$

Several studies have shown an almost immediate decrease in cognitive function when glucocorticoid levels increase to the levels that are seen in animal models of stress. For example, Newcomer and colleagues ${ }^{15}$ found deficits in verbal declarative memory after 4 days of cortisol exposure. These findings not only emphasize the robust and widespread effects of glucocorticoids on the brain, but also suggest a rapid timeline for brain atrophy and hippocampal reorganization.

\section{Reversibility of Cerebral Atrophy in Cushing's Disease}

Cerebral atrophy has been shown to be reversible in patients with Cushing's disease. ${ }^{8}$ Following resection of adrenocorticotropin hormone-secreting pituitary adenomas, hippocampal formation volume has been shown to increase by as much as $10 \% .^{23}$ The increase in hippocampal formation volume correlates with the magnitude of decrease in urinary free cortisol. In addition, improvements in memory correlate with decreases in cortisol levels as well as with increases in hippocampal formation volume. ${ }^{9}$ Age has been identified as a significant factor that influences the speed of recovery. Younger patients regain and sustain their improvement in cognitive functioning more quickly than older subjects. These findings suggest that at least some of the deleterious effects of prolonged hypercortisolemia on cognitive functioning and hippocampal volume are reversible.

In summary, Cushing's disease is associated with hip- pocampal and generalized brain atrophy. Excess glucocorticoids cause retraction and simplification of dendrites in the hippocampus, and these morphological changes probably account for the hippocampal volume loss. Furthermore, a profound loss of synapses is also seen in Cushing's disease. These findings suggest the possibility that volume measures may be underestimating the change in neural structures. Several mechanisms by which glucocorticoids affect the brain include decreased neurogenesis, glucose utilization, and synthesis of neurotrophic factors as well as increased actions of EAA. We have reviewed the evidence correlating hippocampal atrophy and cognitive deficits and have shown that these effects appear to reversible. Further investigations into the mechanisms by which glucocorticoids affect the brain and peripheral tissues are essential. These mechanistic details may eventually provide targets for preventing or treating the brain atrophy, cognitive impairments, and mood disorders common in patients with Cushing's disease.

\section{References}

1. Bentson J, Reza M, Winter J, Wilson G: Steroids and apparent cerebral atrophy on computed tomography scans. J Comput Assist Tomogr 2:16-23, 1978

2. Brown ES, Woolston JD, Frol A, Bobadilla L, Khan DA, Hanczyc M, et al: Hippocampal volume, spectroscopy, cognition, and mood in patients receiving corticosteroid therapy. Biol Psychiatry 55:538-545, 2004

3. Brunetti A, Fulham MJ, Aloj L, De Souza B, Nieman L, Oldfield $\mathrm{EH}$, et al: Decreased brain glucose utilization in patients with Cushing's disease. J Nucl Med 39:786-790, 1998

4. Cereseto M, Reinés A, Ferrero A, Sifonios L, Rubio M, Wikinski $\mathrm{S}$ : Chronic treatment with high doses of corticosterone decreases cytoskeletal proteins in the rat hippocampus. Eur J Neurosci 24:3354-3364, 2006

5. Chapman C, Tubridy N, Cook MJ, Mitchell PJ, MacGregor LR, Lovelock C, et al: Short-term effects of methylprednisolone on cerebral volume in multiple sclerosis relapses. J Clin Neurosci 13:636-638, 2006

6. Coburn-Litvak PS, Tata DA, Gorby HE, McCloskey DP, Richardson G, Anderson BJ: Chronic corticosterone affects brain weight, and mitochondrial, but not glial volume fraction in hippocampal area CA3. Neuroscience 124:429-438, 2004

7. Gould E, Tanapat P, Rydel T, Hastings N: Regulation of hippocampal neurogenesis in adulthood. Biol Psychiatry 48:715-720, 2000

8. Heinz ER, Martinez J, Haenggeli A: Reversibility of cerebral atrophy in anorexia nervosa and Cushing's syndrome. J Comput Assist Tomogr 1:415-418, 1977

9. Hook JN, Giordani B, Schteingart DE, Guire K, Giles J, Ryan K, et al: Patterns of cognitive change over time and relationship to age following successful treatment of Cushing's disease. J Int Neuropsychol Soc 13:21-29, 2007

10. Kadekaro M, Ito M, Gross PM: Local cerebral glucose utilization is increased in acutely adrenalectomized rats. Neuroendocrinology 47:329-334, 1988

11. Koide T, Wieloch TW, Siesjö BK: Chronic dexamethasone pretreatment aggravates ischemic neuronal necrosis. J Cereb Blood Flow Metab 6:395-404, 1986

12. McEwen BS: Glucocorticoids, depression, and mood disorders: structural remodeling in the brain. Metabolism 54 (5 Suppl):20-23, 2005

13. McEwen BS: Stress and hippocampal plasticity. Annu Rev Neurosci 22:105-122, 1999

14. Momose KJ, Kjellberg RN, Kliman B: High incidence of cortical atrophy of the cerebral and cerebellar hemispheres in Cushing's disease. Radiology 99:341-348, 1971 
15. Newcomer JW, Selke G, Melson AK, Hershey T, Craft S, Richards K, et al: Decreased memory performance in healthy humans induced by stress-level cortisol treatment. Arch Gen Psychiatry 56:527-533, 1999

16. Okuno T, Ito M, Konishi Y, Yoshioka M, Nakano Y: Cerebral atrophy following ACTH therapy. J Comput Assist Tomogr 4:20-23, 1980

17. Sapolsky RM, Uno H, Rebert CS, Finch CE: Hippocampal damage associated with prolonged glucocorticoid exposure in primates. J Neurosci 10:2897-2902, 1990

18. Sheline YI: Hippocampal atrophy in major depression: a result of depression-induced neurotoxicity? Mol Psychiatry 1:298-299, 1996

19. Simmons NE, Do HM, Lipper MH, Laws ER: Cerebral atrophy in Cushing's disease. Surg Neurol 53:72-76, 2000

20. Sousa N, Madeira MD, Paula-Barbosa MM: Effects of corticosterone treatment and rehabilitation on the hippocampal formation of neonatal and adult rats. An unbiased stereological study. Brain Res 794:199-210, 1998

21. Spiegel W, McGeady SJ, Mansmann HC: Cerebral cortical atrophy and central nervous system (CNS) symptoms in a steroidtreated child with asthma. J Allergy Clin Immunol 89:918-919, 1992

22. Starkman MN, Giordani B, Berent S, Schork MA, Schteingart DE: Elevated cortisol levels in Cushing's disease are associated with cognitive decrements. Psychosom Med 63:985-993, 2001

23. Starkman MN, Giordani B, Gebarski SS, Berent S, Schork MA, Schteingart DE: Decrease in cortisol reverses human hippocampal atrophy following treatment of Cushing's disease. Biol Psychiatry 46:1595-1602, 1999

24. Starkman MN, Schteingart DE: Neuropsychiatric manifestations of patients with Cushing's syndrome. Relationship to cortisol and adrenocorticotropic hormone levels. Arch Intern Med 141: 215-219, 1981
25. Stein BA, Sapolsky RM: Chemical adrenalectomy reduces hippocampal damage induced by kainic acid. Brain Res 473:175-180, 1988

26. Tata DA, Marciano VA, Anderson BJ: Synapse loss from chronically elevated glucocorticoids: relationship to neuropil volume and cell number in hippocampal area CA3. J Comp Neurol 498:363-374, 2006

27. Uno H, Tarara R, Else JG, Suleman MA, Sapolsky RM: Hippocampal damage associated with prolonged and fatal stress in primates. J Neurosci 9:1705-1711, 1989

28. Watanabe Y, Gould E, Cameron HA, Daniels DC, McEwen BS: Phenytoin prevents stress- and corticosterone-induced atrophy of CA3 pyramidal neurons. Hippocampus 2:431-435, 1992

29. Watanabe Y, Gould E, McEwen BS: Stress induces atrophy of apical dendrites of hippocampal CA3 pyramidal neurons. Brain Res 588:341-345, 1992

30. Woolley CS, Gould E, McEwen BS: Exposure to excess glucocorticoids alters dendritic morphology of adult hippocampal pyramidal neurons. Brain Res 531:225-231, 1990

31. Zhou J, Zhang F, Zhang Y: Corticosterone inhibits generation of long-term potentiation in rat hippocampal slice: involvement of brain-derived neurotrophic factor. Brain Res 885:182-191, 2000

Manuscript submitted June 19, 2007.

Accepted July 13, 2007.

Address correspondence to: Chirag G. Patil, M.D., Department of Neurosurgery, Stanford University Hospital, 300 Pasteur Drive, Room R281, MC5327, Stanford, California 94305. Email: chiragpatil@gmail.com. 